\title{
Designing to Support Reflection on Values \& Practices to Address Online Disinformation
}

\section{Ahmer Arif}

Human Centered Design \& Engineering,

University of Washington

Seattle, WA 98105, USA

ahmer@uw.edu

\section{Abstract}

This research examines crowd and algorithm driven information flows in our current social media ecosystem to understand and address some of the instabilities fueling online misinformation and disinformation. The work combines empirical analysis of social media data, alternative news websites, interviews, and a design intervention to provide insights that are useful for challenging the muddled thinking, passive acquiescence and rote behaviors that give oxygen to online disinformation. Four interrelated studies are synthesized to further our understanding about online disinformation and how we might cultivate new media practices that can aid us in coping under rapidly evolving and challenging conditions.

Permission to make digital or hard copies of part or all of this work for personal or classroom use is granted without fee provided that copies are not made or distributed for profit or commercial advantage and that copies bear this notice and the full citation on the first page. Copyrights for third-party components of this work must be honored. For all other uses, contact the Owner/Author. CSCW '18 Companion, November 3-7, 2018, Jersey City, NJ, USA

(c) 2018 Copyright is held by the owner/author(s).

ACM ISBN 978-1-4503-6018-0/18/11.

https://doi.org/10.1145/3272973.3272974

\section{Keywords}

Disinformation, Misinformation, Social Media, Learning

\section{Introduction}

As CSCW researchers continue to explore the civic potential of social media, we must simultaneously grapple with how these platforms have enabled the widespread dissemination of rumors, conspiracy theories and propaganda. My research addresses this need by offering three contributions. First, it makes empirical and conceptual contributions towards understanding how online misinformation (i.e. unverified or false narratives) and disinformation (i.e. intentional misinformation that fosters doubt and division) spread and engage social media audiences. I do this by closely examining: a) online rumoring behaviors of social media users across two separate breaking news events; and b) some of the salient discursive and network properties of two separate online disinformation campaigns.

Second, to advance our understanding of how online disinformation appeals to our emotional and social reasoning, I put forward a theoretical contribution by placing the grounded understanding I have developed around my empirical data into conversation with ideas from adult and moral psychology [4], [5]. My intention is to bring certain diffuse concepts into focus to help us see how state affiliated disinformation campaigns play 
upon our existing moral and epistemological differences.

Finally, the work I've introduced above is informing and motivating a design contribution. I have an ongoing project in which I am conducting research through design to deliver practical implications around how we might create a certain kind of education to help address networked misinformation and disinformation.

I have become convinced that if people are going to use and shape social media for cooperative civic purposes to reliably and effectively promote human flourishing, then we will need to be not just any kind of people, but people with particular civic characters, motivations and capacities (e.g. have a discerning respect for truth, forbearance and a willingness to engage in reflective self-examination). Consequently, I have designed and deployed learning experiences that Study 1 Study 2 Study 3 Study 4 2020 about what it means to act rightly in relation to technology and other people [10]. Pedagogically, it extends an approach that was developed to support learners with noticing and reflecting on their own media practices and values [6].

\section{Background on Online Disinformation}

Disinformation refers to the intentional spread of inaccurate information to degrade people's decisionmaking capabilities. Although rumors, conspiracy theories and political propaganda are not new, their intersection with social media are not well understood.
For instance, we do not understand how disinformation takes advantage of the networked media landscape to condition and take advantage of our new media practices and habits. This has become a more urgent consideration since announcements made by social media companies in 2017 have acknowledged that their platforms have become a point of access for disinformation campaigns. Russia, in particular, has been singled out for conducting an "information war" designed to rob people of agency by miring them with doubt and confusion [7]. Importantly, the goal of disinformation is not necessarily to alter beliefs, but to strategically direct discourse in ways that "kill the possibility of debate and a reality-based politics" [7]. Social media is an effective tool for waging this type of 'soft' warfare because it can reach people across the world and play on people's habits and practices around these tools to produce emergent and self-reinforcing effects. Simultaneously, trying to gatekeep 'bad' information on these platforms runs the risk of undermining the free speech that is highly valued by liberal democracies.

Grappling with online disinformation will thus require concurrently maneuvering around technology, policy, and education in ways that are not just about reaffirming existing arrangements, but also expanding and reimagining them. For instance, giving people a better social media platform is unlikely to 'solve' the 'problem' of online disinformation if the platform's affordances and values are shaped by the same political conditions as the problem. Similarly, media scholars like danah boyd have noted how relying on traditional media literacy to solve 'fake news' could prove counterproductive by exacerbating both existing epistemological differences and doubt in our 


\section{Example Activity from Study 4: Repulsions}

Learners sought to understand how their own emotional makeup and values triggered certain reading habits in them when they encountered information from sources they had moral differences with.

With careful scaffolding, each learner repeatedly engaged with a news item of their choice that that was repulsive and/or troubling to them (e.g. some learners selfselected the State of the Union address). Learners carefully reflected on their flow of emotions over time and some tried to empathize with those who they imagined to be the media's target audience. Each learner described that this activity was challenging but also immensely rewarding because it helped them learn something about themselves -e.g. certain habits, thoughts or emotions that had previously gone unnoticed. information intermediaries [3]. Rather, she calls for helping people "recognize their own fault lines" rather than just the fault lines of the media landscape around them. This perspective aligns with a growing body of research which suggests that reflective and analytical thinking can reduce conspiratorial ideation and belief in 'fake news' [e.g. [8].

\section{Proposed Research}

At a high-level this research asks: How can we help people cope with the challenging conditions that give rise to online misinformation and disinformation? To approach a more detailed understanding of this complex space, I ask three sub-questions:

1) How are state-affiliated actors spreading disinformation on social media?

2) How do members of the online crowd reflect upon and make sense of their own individual actions when they spread and correct false information on social media?

3) How might we support people in being more informed, intentional and reflective with respect to online misinformation and their own new media practices?

\section{Studies}

The research consists of four separate studies that were carried out sequentially (although not in the order presented here). Studies $1 \& 3$ have been reported on in part in previously published work [2], [9]. Study 2 has been accepted for submission [[1]] while Study 4 represents ongoing work. Moving forward, I will expand and further synthesize previous findings, complete study 4 , and tie findings together into a larger framework.
Study 1: "Questioning More" and Alternative Media To address RQ1, I study how a diverse network of alternative media websites propagate narratives regarding The White Helmets, a volunteer humanitarian group working in the Syrian conflict zone [9]. By combining network and content analyses, this work illuminates how these websites - which are integrated with Russian government-funded media - draw together very different audiences (and perhaps different values) into a shared story that The White Helmets are terrorists and a propaganda construct propped up by Western imperialists. One of the points I want to use my dissertation to elaborate upon involves how these websites deploy the tools and rhetoric of critical thinking to invite readers to write off entire reams of human expertise and experience (e.g. western journalism and academia).

\section{Study 2: Examining Russian Troll Activity in} \#BlackLivesMatter

To further investigate RQ1, Study 2 examines how Russian government agents utilized Twitter and other platforms to influence a highly charged conversation that linked police violence in America during 2016 to the \#BlackLivesMatter movement [1]. The findings from this work - again based on network and content analyses - show that these disinformation actors did not limit themselves to a single "side" of the online conversation. Rather, they cultivated personas - that were all but indistinguishable from other participants in these spaces - to appeal to the values of both rightleaning anti-\#BlackLivesMatter voices and politically left-leaning pro-\#BlackLivesMatter groups. In my dissertation, I intend to bring these findings into conversation with ideas from moral psychology [4]. 


\section{Example Activity from Study 4: Critical Friend Conversations}

Learners both designed for and leveraged technology to explore ways of engaging with the news that afforded more peer-support and opportunities to practice "slow" thinking.

Learners tried out a tool that was prototyped using Google Docs to engage in 16 semistructured pair-wise chats around the news. In these chats, learners either played the as readers or as critical friends. Readers brought in some information about current events that they wanted to spend time thinking about. Critical friends on the other hand, acted as supportive listeners and asked provocative questions, providing data to be examined through another lens. Learners improvised these questions during their chats, with the aid of a handbook that they had coconstructed.
Study 3: A Closer Look at the Self-Correcting Crowd Assessing RQ2, Study 3 analyzes Twitter data to surface different patterns of online rumoring and correcting behavior and interviews 15 Twitter users that exhibited those behaviors [2]. This work provides two viewpoints on the dynamics of online misinformation: an etic understanding of how unverified and false information spreads at the aggregate level; and a more emic one regarding how responsibility for verifying and correcting information can be positioned during breaking news events. Within my dissertation, I intend to explain how this project informed and evolved into the much larger research effort described here.

Study 4: Designing Learning Experiences Around Online Disinformation and Emotional Reasoning

To address RQ3, I am analyzing data from designing and deploying learning experiences as probes with senior undergraduate and masters students. By taking a Research through Design approach, I am surfacing practical insights that are useful for helping others (1) unpack 'fake news' from a sociotechnical perspective; (2) notice their own habits and emotions while consuming news in online settings; and (3) explore what it could be like to step outside of those habits by taking in information in a slower, more mindful way.

\section{References}

[1] Ahmer Arif, Leo Graiden Stewart, and Kate Starbird. 2018. Acting the Part: Examining Information Operations Within \#BlackLivesMatter Discourse. To appear in Proceedings of the 2018 Conference on Computer Supported Cooperative Work and Social Computing (CSCW '18). ACM, New York, NY.

[2] Ahmer Arif, John J. Robinson, Stephanie A. Stanek, Elodie S. Fichet, Paul Townsend, Zena Worku, and Kate
Starbird. 2017. A closer look at the self-correcting crowd: Examining corrections in online rumors. In Proceedings of the 2017 ACM Conference on Computer Supported Cooperative Work and Social Computing (CSCW '17). ACM, New York, NY, 155-168.

[3] danah boyd. 2018. You Think You Want Media Literacy... Do You?. Retrieved April 10th, 2018 from https://points.datasociety.net/you-think-you-wantmedia-literacy-do-you-7cad6af18ec2

[4] Jonathan Haidt. 2012. The righteous mind: Why good people are divided by politics and religion. Vintage.

[5] Robert Kegan. 1994. In over our heads: The mental demands of modern life. Harvard University Press.

[6] David M. Levy. 2016. Mindful tech: How to bring balance to our digital lives. Yale University Press.

[7] Peter Pomerantsev and Michael Weiss. 2014. The menace of unreality: How the Kremlin weaponizes information, culture and money. New York: Institute of Modern Russia

[8] Gordon Pennycook and David G. Rand. 2017. Who falls for fake news? The roles of analytic thinking, motivated reasoning, political ideology, and bullshit receptivity. Yale University.

[9] Kate Starbird, Ahmer Arif, Tom Wilson, Katherine Van Koevering, Katya Yefimova \& Daniel Scarnecchia. 2018. Ecosystem or Echo-System? Exploring Content Sharing across Alternative Media Domains. Forthcoming in Proceedings of the 12th Internationsl AAAI Conference on Web and Social Media (ICWSM-18)

[10] Shannon Vallor. 2016. Technology and the virtues: A philosophical guide to a future worth wanting. Oxford University Press. 